\title{
Management of orthostatic intolerance in children: the state of the art
}

\author{
Chun-Yan $\mathrm{TaO}^{1,2} \cdot$ Hong-Fang Jin ${ }^{1,2} \cdot$ Jun-Bao $\mathrm{Du}^{1,3}$ \\ Received: 5 August 2019 / Accepted: 9 December 2019 / Published online: 7 January 2020 \\ (c) Children's Hospital, Zhejiang University School of Medicine 2020
}

Orthostatic intolerance (OI) is characterized by symptoms and signs of dizziness, headache, visual difficulties, a loss of consciousness, nausea, abdominal discomfort, pallor, tachycardia or hypotension; and $\mathrm{OI}$ is primarily elicited by a rapid postural change from supine to standing or prolonged quiet standing which can be resolved by lying down [1,2]. About $15 \%$ of children and adolescents are affected by OI, with the median peak age of the first OI onset at 15 years [3]. OI accounts for a noticeable proportion of emergency department visits. Deliberately or indeliberately, the injection of normal saline always plays a role, which "rescues" the patients. Single or occasional episodes of OI are often overlooked by some patients; and children with recurrent OI symptoms often experience depression, and have diminished quality of life [4]. Therefore, understanding the pathogenesis of $\mathrm{OI}$ is important. The causes of pediatric OI are multifactorial and heterogeneous, and most are benign and defined as "functional disorders". Orthostatic hypotension $(\mathrm{OH})$, postural tachycardia syndrome (POTS) and vasovagal syncope (VVS) are commonly defined as different hemodynamic responses to orthostatic challenge, which are listed in the category of "functional disorders" [5, 6]. Further understanding of these "functional disorders" can not only reduce the burden of mental stress experienced by patients and improve treatment efficacy but also decrease medical expenses. The past two decades have witnessed progress in understanding the pathophysiology of OI and in the development of various treatment options. Herein, we summarized the proposed pathogenesis of OI and evaluated the therapeutic

Hong-Fang Jin

jinhongfang51@126.com

1 Department of Pediatrics, Peking University First Hospital, No. 1, Xi' an-men Street, West District, Beijing 100034, China

2 Research Unit of Clinical Diagnosis and Treatment of Pediatric Syncope and Cardiovascular Diseases, Chinese Academy of Medical Sciences, Beijing, China

3 Key Laboratory Molecular Cardiovascular Science, the Ministry of Education, Peking University, Beijing, China strategies, especially personalized treatment (Fig. 1), to promote optimal treatment strategies for patients with OI.

\section{Physiological response to orthostatic stress}

Upon reaching an upright position, increased gravitational forces cause a central blood volume shift of $500-1000 \mathrm{~mL}$ from the chest into the vessels below the level of the heart [7-9]. The reduced venous return decreases the pulsatile distortion of baroreceptors located in the arterial and cardiopulmonary regions. Then, the signals from these receptors are conveyed to the central nervous system, which regulates sympathetic activation and parasympathetic withdrawal within seconds. Simultaneously, skeletal muscle in the legs propels blood back to the heart. Respiration increases venous return by declining thoracic pressure and ascending intraabdominal pressure during deep inspiration. Consequently, a small increase in the heart rate ( $\sim 20$ beats/min) and the maintenance of the mean blood pressure [a negligible variation in systolic blood pressure and a slight increase in diastolic blood pressure $(\sim 5 \mathrm{~mm} \mathrm{Hg})]$ are observed, and appropriate perfusion to critical organs is retained. Meanwhile, cerebral perfusion is also regulated and maintained by its autoregulation (unchanged cerebral blood flow despite the rational change in blood pressure) [10]. During prolonged quiet standing, in addition to venous pooling, transcapillary filtration of plasma in regions under the diaphragm further decreases the effective circulating blood volume by approximately $15-20 \%$, and after approximately 30 minutes of upright posture, this shift is balanced. A continuous upright position activates neuroendocrine processes such as the renin-angiotensin-aldosterone system to regulate the haemodynamic equilibrium according to the subject's exact volume status [9].

\section{Pathogenesis of orthostatic intolerance}

The pathogenesis of OI is complex and has not yet been fully understood, but some common and universal mechanisms have been widely accepted in the field. Most 


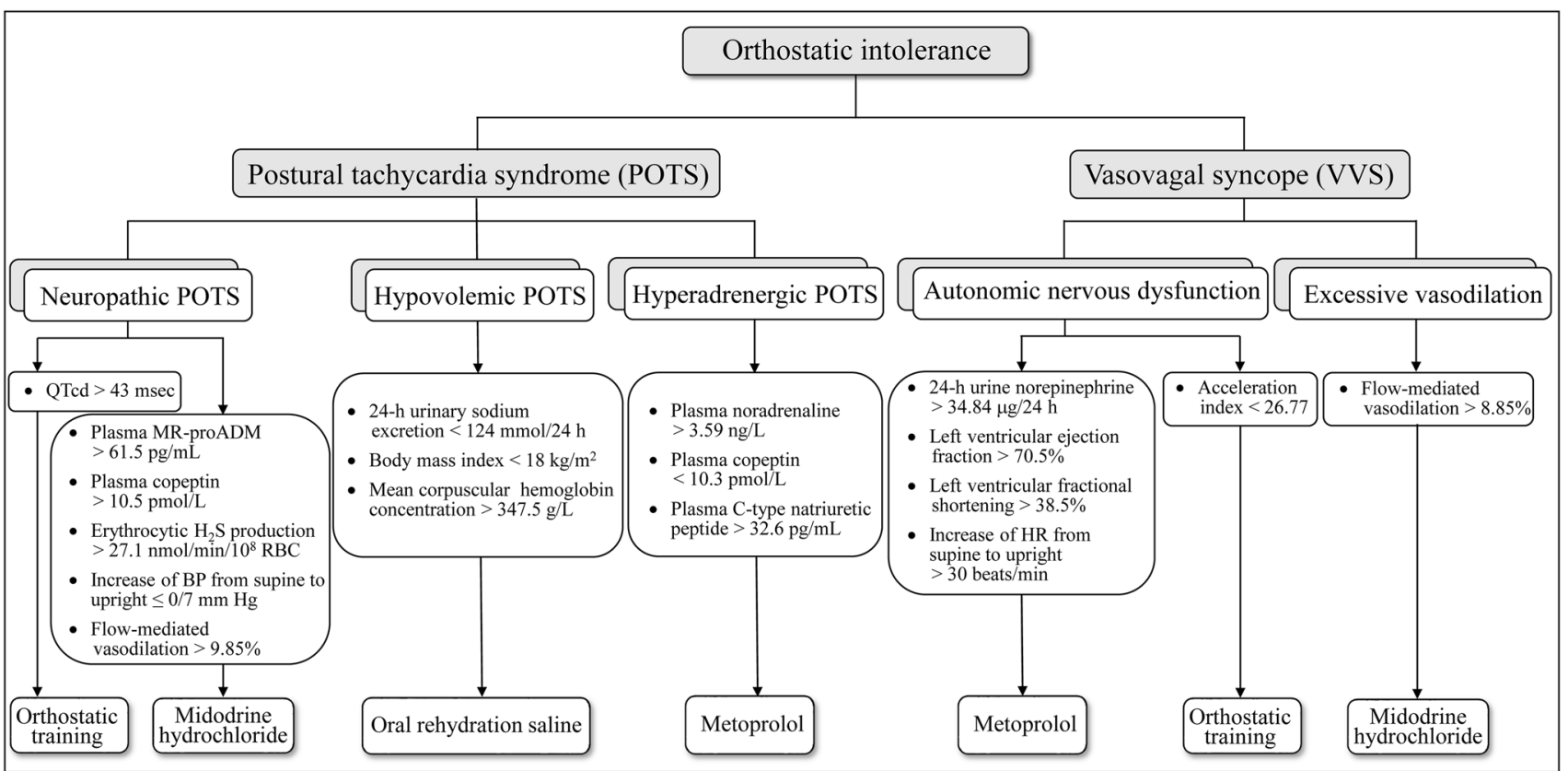

Fig. 1 A schematic diagram showing the individualized therapeutic strategy for paediatric orthostatic intolerance in the literature. QTcd
HR-corrected QT interval dispersion, $M R-p r o A D M$ midregional proadrenomedullin, $B P$ blood pressure, $H R$ heart rate patients with OI have their orthostatic symptoms because of the insufficient central blood volume. Reduced fluid intake, excessive excretion from diarrhoea, vomiting or sweating, and abnormal distribution may be the triggering factors [11, 12]. Abnormal autonomic function has also been reported in a substantial portion of patients with OI. However, the variation among the different types of OI in patients is paradoxical and controversial, as either impaired or over-activated sympathetic function is observed [13, 14]. Another acknowledged mechanism is vasomotor dysfunction from peripheral autonomic denervation or an imbalance between vasodilators and vasoconstrictors, which results in impaired vasoconstriction and/or excessive vasodilation $[15,16]$. About half of patients with POTS are in the hyperadrenergic subgroup, as determined by elevated plasma norepinephrine levels when a patient is upright [17]. Studies suggest that the impaired clearance of intra-synaptic norepinephrine or excessive synthesis of norepinephrine may be the cause $[18,19]$. These patients complain of typical symptoms including palpitations, tremors, pallor, hyperhidrosis or abdominal discomfort when standing. A putative mechanism is autoimmunity. Previous studies identified associations with viral illness, patient histories of systemic autoimmune diseases and an increased prevalence of serum autoantibodies [8]. Moreover, OI maybe genetic because some patients reported a family history of OI and a loss-of-function mutation in the $S L C 6 A 2$ gene encoding the norepinephrine transport receptor was found in one family with POTS $[18,20,21]$.
The pathological mechanisms that contribute to OI are complex and often overlap. Different therapies are required according to different mechanisms. This makes it increasingly difficult to effectively treat patients with a greater variety of the underlying mechanisms of OI. Many patients with OI experience a reduced quality of life. Therefore, it is important to determine effective treatments for OI.

\section{Management of orthostatic hypotension}

$\mathrm{OH}$ is regarded as a poor physiological response to external stimuli. $\mathrm{OH}$ is considered as neurogenic $\mathrm{OH}$ when cuased by autonomic nervous system failure; otherwise it is considered as non-neurogenic $\mathrm{OH}$. It is divided into three categories (classic, delayed and initial $\mathrm{OH}$ ) on the basis of the onset time when blood pressure decreases dramatically [9]. Classic $\mathrm{OH}$ is the main form of pediatric $\mathrm{OH}$, and is defined by a steady decrease in systolic blood pressure by $\geq 20 \mathrm{~mm} \mathrm{Hg}$ and/or diastolic blood pressure by $\geq 10 \mathrm{~mm} \mathrm{Hg}$ within $3 \mathrm{~min}$ utes after standing [22]. Less than 5\% of the young population is affected by $\mathrm{OH}$, but this may be an underestimation because $\mathrm{OH}$ is common in asymptomatic individuals [23]. Tailored interventions should be given once $\mathrm{OH}$ is diagnosed [9, 24]. Importantly, patients should understand the postural pathophysiology and avoid triggers. Conventional treatments for orthostatic symptoms include the completion of physical counter-pressure manoeuvres and rapid water ingestion. In such settings, the use of elastic stockings and 
abdominal binding may be helpful [25]. However, because patients report discomfort and inconvenience with this intervention, it is advised only as needed, such as during an inevitable orthostatic challenge. Pharmacological agents are recommended if symptoms are not controlled by the abovementioned measures. However, drugs such as midodrine hydrochloride, fludrocortisone, and recombinant erythropoietin are infrequently used in the pediatric clinical practice because of a lack of evidence-based researches [10]. Droxidopa effectively treats neurogenic $\mathrm{OH}$ in the elderly population, but it is scarcely considered in pediatric patients due to a low incidence of neurogenic $\mathrm{OH}$ in the youth [26, 27]. Further studies in children and adolescents are needed to determine the roles of pharmacological treatment.

\section{Management of postural tachycardia syndrome}

POTS is another main component of pediatric OI, characterized by chronic day-to-day symptoms and an excessive increase in heart rate when upright (normal supine heart rate and an increase in heart rate $\geq 40$ beats/min within the initial 10 minutes after standing without hypotension) [28-31]. For a long time, the diagnostic criteria of pediatric POTS relied on adult guidelines which recommended a cut-off value of $\geq 30$ beats/min [32]. Cardiovascular regulation in children and adolescents is immature and changes in heart rate and blood pressure with postural change are entirely different from those of adults [33]. In 2015, Zhao et al. conducted a large cross-sectional study and demonstrated a difference between adults and children [6].

Given the complex and overlapping underlying mechanisms for POTS, there is no uniform treatment for all patients with POTS. Regardless of the causes of POTS (partial autonomic neuropathy, hyperadrenergic state, blood volume dysregulation, genetic mutations of norepinephrine transporters, mast cell activation, autoimmunity, deconditioning, etc.), the mainstay effects funnel into three endophenotypes (neuropathic POTS, hypovolemic POTS, and hyperadrenergic POTS) [34, 35].

More than $50 \%$ of patients with POTS have peripheral autonomic denervation [20]. The final effect of peripheral autonomic denervation leads to the excessive pooling of blood in the lower extremities and pelvic and splanchnic organs. Neuropathic POTS is treated by promoting venous return [35]. Abdominal binders and leg compression garments are effective for promoting venous return, but this intervention is challenging for youths because of its inconvenience and uncomfortableness. Orthostatic training, an efficient way to improve autonomic function, plays an important role in treatment due to its noninvasiveness and simplicity. Disappointingly, no consistent positive efficacy has been reported. Lu and colleagues applied HR-corrected QT interval dispersion to indicate autonomic function to distinguish those who would respond more efficiently to orthostatic training. They suggested that patients with a HRcorrected QT interval dispersion $>43 \mathrm{~ms}$ should be treated with orthostatic training because they would be prone to better outcomes [36]. Because of lacking researches on potential adverse effects of medications, nonpharmacological regimens should be considered unless severe symptoms cannot be resolved. Midodrine hydrochloride, a prodrug converted to an active $\alpha 1$-adrenergic agonist, is used in POTS patients based on the rationale of its vasoconstriction. Our team found that three plasma indices including midregional pro-adrenomedullin, copeptin, and erythrocytic hydrogen sulfide production can predict therapeutic reponse to midodrine hydrochloride well; the levels of midregional pro-adrenomedullin $>61.5 \mathrm{pg} / \mathrm{mL}$, copeptin $>10.5 \mathrm{pmol} / \mathrm{L}$, or erythrocytic hydrogen sulfide production $>27.1 \mathrm{nmol} / \mathrm{min} / 10^{8}$ erythrocytes predicted a better response [37-39]. Nonetheless, venepuncture and complex procedures of examination limited their application. To overcome this disadvantage, Deng et al. creatively proposed that changes in blood pressure from supine to standing might in part reflect the state of vascular tone. This was confirmed when they observed a decrease in blood pressure from the supine position to the upright position more often in responders [40]. More directly, Liao and colleagues revealed that flow-mediated vasodilation detected by colour Doppler vascular ultrasound was visually reflective of peripheral vascular resistance and could predict the response of POTS patients treated with midodrine hydrochloride. Midodrine hydrochloride was effective in patients whose flow-mediated vasodilation was $>9.85 \%$ [41]. Importantly, most of these markers can also predict patients' longterm outcomes [42-44].

Hypovolemia is common in pediatric POTS patients. Aerobic reconditioning training is highly recommended as an effective method to augment blood volume. However, low medical adherence greatly limits its effect [35]. Supplementation of fluid and salt is another method used to increase the blood volume, but it is not consistently effective as it can be challenging for patients with a complex pathophysiology to complete the regimen for a high water and salt diet $[11,45]$. Our team investigated several biomarkers to predict patients' responses to fluid and salt supplementation. Twenty-four-hour urinary sodium excretion, body mass index and mean corpuscular hemoglobin concentration were shown to be associated with blood volume to some degree. Those patients with a 24-h urinary sodium excretion $<124 \mathrm{mmol} / 24 \mathrm{~h}$, body mass index $<18 \mathrm{~kg} / \mathrm{m}^{2}$ or mean corpuscular haemoglobin concentration $>347.5 \mathrm{~g} / \mathrm{L}$ showed a positive response to fluid and salt supplement [11, $45,46]$. Furthermore, patients with lower urinary sodium 
excretion likely had a better long-term outcome after an oral rehydration saline intervention [47]. Regarding medications (e.g., fludrocortisone, desmopressin, recombinant erythropoietin, etc.), few studies have been carried out in pediatric patients.

Many POTS patients had high plasma catecholamine levels and excessive sympathetic activation upon standing. We should discontinue any medications which may worsen or precipitate the hyperadrenergic condition. Treatments that suppress sympathetic nervous function or augment parasympathetic nervous function are beneficial. Beta-blockers of adrenergic receptors, the mainstay of sympathetic nervous function suppression, are widely used to treat patients with POTS. However, their controversial therapeutic efficacy highlights a need for more valuable predictive parameters. Our team found that orthostatic plasma norepinephrine level of $>3.59 \mathrm{pg} / \mathrm{mL}$ was an indicator of the effectiveness of metoprolol therapy for POTS in children and adolescents [48]. Even with a relatively high predictive value, this parameter is criticized by many scholars for its sharp variation by stimuli. Our team further found that the response to metoprolol using plasma copeptin and C-type natriuretic peptide can predict the patients' response to metoprolol [49, 50]. Pyridostigmine, which enhances cholinergic activity and activates parasympathetic nervous function, was found to be partly effective in a few small sample-sized populations [51-53].

\section{Management of vasovagal syncope}

VVS is another primary form of OI in children. It is characterized by syncope or pre-syncope and transiently systemic hypotension with or without bradycardia. VVS is responsible for $60-70 \%$ of pediatric cases with syncope [54]. Nearly $80 \%$ of reflex syncopal patients present with prodromes. During a prodrome, countermeasures should be implemented to prevent a syncopal episode [55]. However, countermeasures are not suggested to prevent asystolic VVS because it occurs without prodromes. Under such conditions, loop recording electrocardiography should be considered in addition to normal 24-h Holter EKG monitoring. Beta-adrenergic blockers are the often used therapeutic drug on the basis of an abnormal BezoldJarisch reflex and high levels of plasma epinephrine and norepinephrine in patients with VVS [56]. However, the efficacy of such treatments remains controversial $[57,58]$. Kong et al. recently revealed a noninvasive method for predicting the response of VVS patients who received metoprolol; they found that 24-h urine norepinephrine measurement was valuable for predicting the usefulness of beta-adrenergic blocker treatments with a sensitivity of $70 \%$ and high specificity of $100 \%$ with a cutoff value of $34.84 \mu \mathrm{g} / 24 \mathrm{~h}$ [59]. However, the use of one full day for measurements is not time-effective. Song et al. demonstrated that echocardiogram-derived parameters, e.g., left ventricular ejection fraction and fractional shortening, better predicted the treatment efficacy of beta-adrenergic blockers in VVS children. Both the left ventricular ejection fraction and fractional shortening indirectly represented plasma catecholamine levels with the ability to predict the efficacy of metoprolol [60]. Furthermore, the investigators used a sharp increase of heart rate before the positive response in the head-up tilt test to predict the efficacy of metoprolol for pediatric VVS. Patients whose heart rate increased by 30 beats/min or more had a more favourable prognosis after metoprolol treatment. Despite a high sensitivity and specificity of $81 \%$ and $89 \%$, the utility of measuring heart rate changes in clinics was restricted by the extremely small sample size and the lack of external validation [61]. The role of orthostatic training in treating paediatric VVS patients is disputed due to a lack of consistent efficacy. We conducted a study to explore the acceleration index, which is associated with sympathetic activity, by comparing its value in responders and nonresponders to orthostatic training. The acceleration index was much lower in the former than in the latter. An acceleration index value $<26.77$ was used to predict positive outcomes [62].

Excessive vasodilation is criticized for its participation in the genesis of VVS. Flow-mediated vasodilation, as discussed above, also showed predictive qualities, with a sensitivity of $90 \%$ and a specificity of $80 \%$ when a flow-mediated vasodilation cutoff value of $8.85 \%$ was used [63].

\section{Conclusions}

OI significantly diminishes the quality of life in many young individuals, and is studied closely by pediatricians. Unfortunately, its exact pathogenesis remains unclear, and treatment strategies still need to be optimized. Present indicators fail to sufficiently and accurately predict the therapeutic efficacy and long-term prognosis. Heterogeneous manifestations, obscure and likely overlapping mechanisms, as well as confounding factors, pose challenges in effective treatment. In addition, comorbidities such as joint hypermobility, migraines, fibromyalgia, and chronic nausea have complicated relations with OI, and their treatments are rather difficult. Future studies are required to elucidate the diverse mechanisms underlying OI, to determine the relationship between comorbidities and OI, and to improve treatment effectiveness. Certainly, well-designed and large sample-sized studies are needed to find evidence-based treatment strategies for improving patient care. 
Author contribution TCY had the primary idea and wrote the draft. JHF and DJB revised the manuscript and had a final approval of it. All authors have read and approved the final manuscript and assumed full responsibility for its contents.

Funding This work was supported by Science and Technology Program of Beijing (No. Z171100001017253), Peking University Clinical Scientist Program (No. BMU2019LCKXJ001), Peking University Medicine Fund of Fostering Young Scholars' Scientific and Technological Innovation, Peking University Clinical Medicine Plus X-Young Scholars Project (No. PKU2019LCXQ011), and Fundamental Research Funds for the Central Universities.

\section{Compliance with ethical standards}

Ethical approval Not needed.

Conflict of interest The authors declare that they have no conflict of interest.

\section{References}

1. Stewart JM. Update on the theory and management of orthostatic intolerance and related syndromes in adolescents and children. Expert Rev Cardiovasc Ther. 2012;10:1387-99.

2. Stewart JM, Boris JR, Chelimsky G, Fischer PR, Fortunato JE, Grubb BP, et al. Pediatric disorders of orthostatic intolerance. Pediatrics. 2018;141:1673.

3. Kenny RA, Bhangu J, King-Kallimanis BL. Epidemiology of syncope/collapse in younger and older western patient populations. Prog Cardiovasc Dis. 2013;55:357-63.

4. Pederson CL, Brook JB. Health-related quality of life and suicide risk in postural tachycardia syndrome. Clin Auton Res. 2017;27:75-81.

5. Hamrefors V, Spahic JM, Nilsson D, Senneby M, Sutton R, Melander O, et al. Syndromes of orthostatic intolerance and syncope in young adults. Open Heart. 2017;4:e000585.

6. Zhao J, Han Z, Zhang X, Du S, Liu AD, Holmberg L, et al. A cross-sectional study on upright heart rate and $\mathrm{BP}$ changing characteristics: basic data for establishing diagnosis of postural orthostatic tachycardia syndrome and orthostatic hypertension. BMJ Open. 2015;5:e007356.

7. Jardine DL, Wieling W, Brignole M, Lenders JWM, Sutton R, Stewart J. The pathophysiology of the vasovagal response. Heart Rhythm. 2018;15:921-9.

8. Bryarly M, Phillips LT, Fu Q, Vernino S, Levine BD. Postural orthostatic tachycardia syndrome: JACC focus seminar. J Am Coll Cardiol. 2019;73:1207-28.

9. Ricci F, De Caterina R, Fedorowski A. Orthostatic hypotension: epidemiology, prognosis, and treatment. J Am Coll Cardiol. 2017;66:848-60.

10. Stewart JM. Common syndromes of orthostatic intolerance. Pediatrics. 2013;131:968-80.

11. Li H, Wang Y, Liu P, Chen Y, Feng X, Tang C, et al. Body mass index (BMI) is associated with the therapeutic response to oral rehydration solution in children with postural tachycardia syndrome. Pediatr Cardiol. 2016;37:1313-8.

12. Ruzieh M, Grubb BP. Orthostatic intolerance and postural tachycardia syndrome: new insights into pathophysiology and treatment. Herzschrittmacherther Elektrophysiol. 2018;29:183-6.
13. Peltier AC, Garland E, Raj SR, Sato K, Black B, Song Y, et al. Distal sudomotor findings in postural tachycardia syndrome. Clin Auto Res. 2010;20:93-9.

14. Tao C, Tang C, Chen S, Jin H, Du J. Autonomic nervous function in vasovagal syncope of children and adolescents. Neurosci Bull. 2019;35:937-40.

15. Bai W, Chen SY, Jin HF, Du JB. Vascular dysfunction of postural tachycardia syndrome in children. World J Pediatr. 2018;14:13-7.

16. Santini L, Capria A, Brusca V, Violo A, Smurra F, Scarfò I, et al. An increased endothelial-independent vasodilation is the hallmark of the neurally mediated syncope. Clin Cardiol. 2012;35:107-10.

17. Grubb BP. Postural tachycardia syndrome. Circulation. 2008;117:2814-7.

18. Shannon JR, Flattern NL, Jordan J, Jacob G, Black BK, Biaggioni I, et al. Orthostatic intolerance and tachycardia associated with norepinephrinetransporter deficiency. N Engl J Med. 2000;342:541-9.

19. Bayles R, Harikrishnan KN, Lambert E, Baker EK, Agrotis A, Guo L, et al. Epigenetic modification of the norepinephrine transporter gene in postural tachycardia syndrome. Arterioscler Thromb Vasc Biol. 2012;32:1910-6.

20. Thieben MJ, Sandroni P, Sletten DM, Benrud-Larson LM, Fealey RD, Vernino S, et al. Postural orthostatic tachycardia syndrome: the Mayo clinic experience. Mayo Clin Proc. 2007;82:308-13.

21. Goodman BP. Evaluation of postural tachycardia syndrome (POTS). Auton Neurosci. 2018;215:12-9.

22. Freeman R, Abuzinadah AR, Gibbons C, Jones P, Miglis MG, Sinn DI. Orthostatic hypotension JACC state-of-the-art review. J Am Coll Cardiol. 2018;72:1294-309.

23. Fagard RH, De Cort P. Orthostatic hypotension is a more robust predictor of cardiovascular events than nighttime reverse dipping in elderly. Hypertension. 2010;56:56-61.

24. Wieling W, van Dijk N, Thijs RD, de Lange FJ, Krediet CT, Halliwill JR. Physical countermeasures to increase orthostatic tolerance. J Inter Med. 2015;277:69-82.

25. Podoleanu C, Maggi R, Brignole M, Croci F, Incze A, Solano $\mathrm{A}$, et al. Lower limb and abdominal compression bandages prevent progressive orthostatic hypotension in elderly persons: a randomized single-blind controlled study. J Am Coll Cardiol. 2006;48:1425-32.

26. Eschlböck S, Wenning G, Fanciulli A. Evidence-based treatment of neurogenic orthostatic hypotension and related symptoms. J Neural Transm. 2017;124:1567-605.

27. Tanaka H, Yamaguchi H, Mino M. The effects of the noradrenaline precursor, L-threo-3,4-dihydroxyphenylserine, in children with orthostatic intolerance. Clin Auton Res. 1996;6:189-93.

28. Wang C, Li Y, Liao Y, Tian H, Huang M, Dong X, et al. 2018 Chinese Pediatric Cardiology Society (CPCS) guideline for diagnosis and treatment of syncope in children and adolescents. Sci Bull. 2018;63:1558-64.

29. Stewart J. A new guideline for diagnosis and treatment of syncope in children and adolescents that stimulates further thought and discussion. Sci Bull. 2018;63:1527-8.

30. Tao C, Liu X, Zhang C, Chen Y, Huang Y. Comments on 2018 CPCS guideline for diagnosis and treatment of syncope in children and adolescents. Sci Bull. 2019;64:291-2.

31. Xu W, Wang T. Diagnosis and treatment of syncope in pediatric patients: a new guideline. Sci Bull. 2019;64:357-8.

32. Chinese Pediatric Cardiology Society, Editorial Board of Chinese Journal of Pediatrics. Guideline of diagnosis of syncope in children. Chin J Pediatr. 2009;47:99-101.

33. Janz KF, Dawson JD, Mahoney LT. Predicting heart growth during puberty: the muscatine study. Pediatrics. 2000;105:E63.

34. Raj SR. Postural tachycardia syndrome (POTS). Circulation. 2013;127:2336-42. 
35. Mar PM, Raj SR. Postural orthostatic tachycardia syndrome: mechanisms and new therapies. Annu Rev Med. 2019. https:// doi.org/10.1146/annurev-med-041818-011630.

36. Lu W, Yan H, Wu S, Chen S, Xu W, Jin H, et al. Electrocardiography-derived predictors for therapeutic response to treatment in children with postural tachycardia syndrome. J Pediatr. 2016;176:128-33.

37. Zhang F, Li X, Ochs T, Chen L, Liao Y, Tang C, et al. Midregional pro-adrenomedullin as a predictor for therapeutic response to midodrine hydrochloride in children with postural orthostatic tachycardia syndrome. J Am Coll Cardiol. 2012;60:315-20.

38. Zhao J, Tang C, Jin H, Du J. Plasma copeptin and therapeutic effectiveness of midodrine hydrochloride on postural tachycardia syndrome in children. J Pediatr. 2014;165(290):e1.

39. Yang J, Zhao J, Du S, Liu D, Fu C, Li X, et al. Postural orthostatic tachycardia syndrome with increased erythrocytic hydrogen sulfide and response to midodrine hydrochloride. J Pediatr. 2013;163:e2.

40. Deng W, Liu Y, Liu AD, Holmberg L, Ochs T, Li X, et al. Difference between supine and upright blood pressure associates to the efficacy of midodrine on postural orthostatic tachycardia syndrome (POTS) in children. Pediatr Cardiol. 2014;35:719-25.

41. Liao Y, Yang J, Zhang F, Chen S, Liu X, Zhang Q, et al. Flowmediated vasodilation as a predictor of therapeutic response to midodrine hydrochloride in children with postural orthostatic tachycardia syndrome. Am J Cardiol. 2013;112:816-20.

42. Li H, Zhang F, Wang Y, Liu P, Zhang C, Feng X, et al. Predictive value of baseline plasma midregional fragment of pro-adrenomedullin level on long-term outcome of postural tachycardia syndrome children treated with midodrine hydrochloride. Chin J Cardiol. 2015;43:507-10.

43. Sun C, Li H, Yang J, Li X, Wang Y, Liu P, et al. Long-term follow-up of POTS children treated with midodrine hydochloride: predictive significance of baseline erythrocytic hydrogen sulfide production level. Chin J Pract Pediatr. 2016;31:590-3.

44. Li HX, Deng WJ, Zhang CY, Jin HF, Du JB. Predictive value of upright blood pressure change for long-term prognosis of children with postural tachycardia syndrome treated with midodrine hydrochloride. Chin J Pediatr. 2016;54:519-22.

45. Lu W, Yan H, Wu S, Xu W, Jin H, Du J. Hemocytometric measures predict the efficacy of oral rehydration for children with postural tachycardia syndrome. J Pediatr. 2017;187:220-4.

46. Zhang Q, Liao Y, Tang C, Du J, Jin H. Twenty-four-hour urinary sodium excretion and postural orthostatic tachycardia syndrome. J Pediatr. 2012;161:281-4.

47. Li J, Zhang Q, Liao Y, Zhang C, Du J. Clinical value of 24-hour urinary sodium determination in children with postural tachycardia syndrome. Chin J Pediatr. 2015;53:203-7.

48. Zhang Q, Chen X, Li J, Du J. Orthostatic plasma norepinephrine level as a predictor for therapeutic response to metoprolol in children with postural tachycardia syndrome. J Transl Med. 2014;12:249.

49. Zhao J, Du S, Yang J, Lin J, Tang C, Du J, et al. Usefulness of plasma copeptin as a biomarker to predict the therapeutic effectiveness of metoprolol for postural tachycardia syndrome in children. Am J Cardiol. 2014;114:601-5.
50. Lin J, Han Z, Li H, Chen SY, Li X, Liu P, et al. Plasma C-type natriuretic peptide as a predictor for therapeutic response to metoprolol in children with postural tachycardia syndrome. PLoS ONE. 2015;10:e0121913.

51. Filler G, Gow RM, Nadarajah R, Jacob P, Johnson G, Zhang $\mathrm{Y}$, et al. Pharmacokinetics of pyridostigmine in a child with postural tachycardia syndrome. Pediatrics. 2006;118:e1563-e15681568.

52. Kanjwal K, Karabin B, Sheikh M, Elmer L, Kanjwal Y, Saeed B, et al. Pyridostigmine in the treatment of postural orthostatic tachycardia: a single-center experience. Pacing Clin Electrophysiol. 2011;34:750-5.

53. Kimpinski K, Figueroa JJ, Singer W, Sletten DM, Iodice V, Sandroni P, et al. A prospective, 1-year follow-up study of postural tachycardia syndrome. Mayo Clin Proc. 2012;87:746-52.

54. Romme JJ, van Dijk N, Boer KR, Dekker LR, Stam J, Reitsma JB, et al. Influence of age and gender on the occurrence and presentation of reflex syncope. Clin Auto Res. 2008;18:127-33.

55. Chen L, Zhang Q, Ingrid S, Chen J, Qin J, Du J. Aetiologic and clinical characteristics of syncope in Chinese children. Acta Paediatr. 2007;96:1505-10.

56. Raj SR, Faris PD, Semeniuk L, Manns B, Krahn AD, Morillo CA, et al. Rationale for the assessment of metoprolol in the prevention of vasovagal syncope in aging subjects trial (POST5). Am Heart J. 2016;174:89-94.

57. Brignole M, Moya A, de Lange FJ, Deharo JC, Elliott PM, Fanciulli A, et al. 2018 ESC Guidelines for the diagnosis and management of syncope. Eur Heart J. 2018;39:1883-948.

58. Shen WK, Sheldon RS, Benditt DG, Cohen MI, Forman DE, Goldberger ZD, et al. 2017 ACC/AHA/HRS guideline for the evaluation and management of patients with syncope: a report of the American College of Cardiology/American Heart Association Task Force on Clinical Practice Guidelines and the Heart Rhythm Society. Heart Rhythm. 2017;14:e155-217.

59. Kong Q, Yang X, Cai Z, Pan Y, Wang W, Liu M, et al. Twentyfour-hour urine NE level as a predictor of the therapeutic response to metoprolol in children with recurrent vasovagal syncope. Ir J Med Sci. 2019;188:1279-87.

60. Song J, Li H, Wang Y, Liu P, Li X, Tang C, et al. Left ventricular ejection fraction and fractional shortening are useful for the prediction of the therapeutic response to metoprolol in children with vasovagal syncope. Pediatr Cardiol. 2018;39:1366-72.

61. Zhang QY, Du JB, Zhen JL, Li WZ, Wang YL. Hemodynamic changes during head-up tilt test and predictive value thereof in predicting the efficacy of metoprolol therapy in children with vasovagal syncope. Natl Med J China. 2007;87:1260-2.

62. Tao C, Li X, Tang C, Jin H, Du J. Acceleration index predicts efficacy of orthostatic training on vasovagal syncope in children. J Pediatr. 2019;207:54-8.

63. Zhang FW, Liao Y, Li XY, Chen L, Jin HF, Du JB. The predictive value of flow-mediated vasodilation on therapeutic efficacy of midodrine hydrochloride for vasovagal syncope in children. Chin J Prac Pediatr. 2012;27:102-5.

Publisher's Note Springer Nature remains neutral with regard to jurisdictional claims in published maps and institutional affiliations. 